\title{
Selective Ring-opening Fluorination of Epoxide: An Efficient Synthesis of 2'-C-Fluoro-2'-C-methyl Carbocyclic Nucleosides
}

\author{
Lian Jin Liu, ${ }^{\dagger}$ Si Wouk Kim, Wonjae Lee, and Joon Hee Hong
}

\author{
BK21-Project Team, College of Pharmacy, Chosun Lnwersity, Kwangin 501-759, Korea. "E-mail: honghactchosun.ac.kr \\ - Department of Enirommental Engineering, BK2I Team for Biohvdrogen Production. Chosun Lniversity, \\ Kwangiu 501-759, Korea \\ Received September 5, 2009. Accepted October 16, 2009
}

\begin{abstract}
An efficient sy'nthetic route of novel $2^{\prime}(\alpha)-C$-fluoro-2' $(\beta)-C$-methyl carbocyclic nucleoside analogues is described. The key fluorinated untennediate 7 was prepared from the epoxide intemediate 5 via selective ring-opening of epoxide. Coupling of 7 with nucleosidic bases under the Mitsunobu reactions followed by deprotection afforded the target carbocyclic nucleoside analogues. The synthesized compounds were evaluated as inhibitors of the hepatitis $\mathrm{C}$ virus (HCV) in Huh-7 cell line in vitro.
\end{abstract}

Key Words: Fluorohydrin. Carbocyclic nucleoside. Anti-HCV agent. Mitsunobu reaction

\section{Introduction}

The infection of hepatitis $\mathrm{C}$ virus $(\mathrm{HCV})^{1}$. accounts for many hepatitis cases worldwide and is also strongly associated with the development of cirrhosis and hepatocellular carcinoma. The current standard therapy for chronic HCV infection is interferon- $\alpha$ in combination with ribavirin. which is inadequate because of the low response rates as well as its side effects.

The molecular virology of $\mathrm{HCV}$ has led to the identification of a mumber of antiviral molecular targets. including the NS5B RNA-dependent RNA polymerase. Inhibition of this enzyme inhibits HCV replication. making this enzy'me a crucial target for new anti-HCV agents. Many nucleoside analogues have been evaluated as anti-HCV agents. ${ }^{3}$ These nucleosides are incorporated into proviral RNA like a substrate after being converted to their corresponding triphosphates and act as chain teminitors. Modification in the vicinity of the 2'-hy'drosy of the ribose in natural ribonucleosides can produce effective RNA chain terminators. ${ }^{4}$ For example replacement of the $2^{2}$-hydrogen of natural ribonucleosides with a methyl group yields compounds with excellent chain-terminating properties. Among them. $2^{\prime}-C$-methyladenosine 1 and $2^{\prime}-C$-methylcytidine 2 are potent anti$\mathrm{HCV}$ agents in clinical trials (Figure 1). More recently. $2^{\prime}-\mathrm{C}$ fluoro-2'-C-methylcytidine 3 was designed as a hepatitis $C$ virus RNA-dependant RNA poly merase (HCV RdRp) inhibitor and showed better inhibitory activity in the HCV replicon assay than 2'-C-methy lcytidine, with low cellular toxicity

Carbocyclic nucleosides are a group of compounds structurally analogous to natural and synthetic nucleosides in which the furanose oxygen has been replace by a methylene group. This replacement changes the furanose ring into a cyclopentane. The expected similarity in bond lengths and bond angles of the tetrahydrofuran and cyclopentane rings allows these analogues to behave as substrates or inhibitors of the enzymes in living cells. Therefore. the carbocyclic nucleosides possess a wide range of biological activities such as antiviral and antitumor effects.
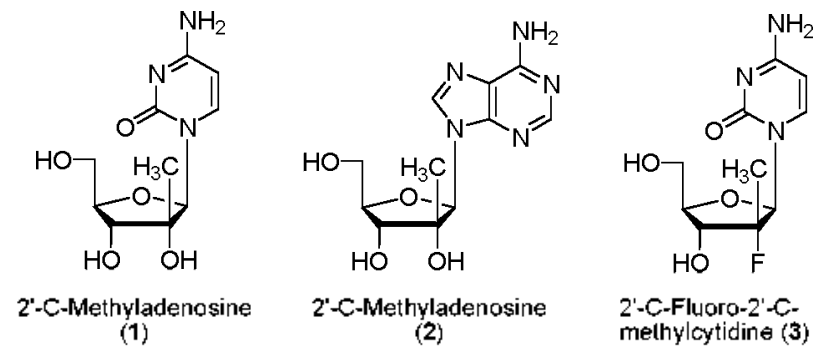

Figure 1. Structure of potent anti-HCV agents.
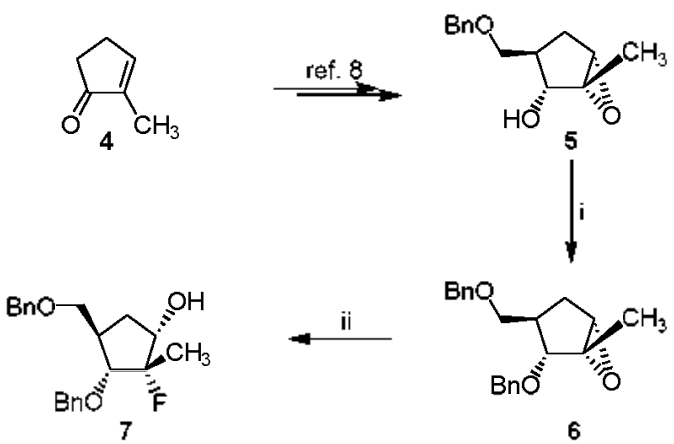
2'-C-Fluoro-2'-C-
melhylcytidine (3)

\section{Scheme 1. Synthesis of fluorinated key intermediate 7. Reagents: i) $\mathrm{BnBr}, \mathrm{NaH}, \mathrm{DMF} ;$ ii) $47 \% \mathrm{HF},\left(\mathrm{NH}_{4}\right)_{2} \mathrm{SiF}, \mathrm{CsF}$}

Based on this information we designed fluorinated analogues of carbocyclic nucleosides as anti-HCV agents, focusing on the modification of the 2'-position of the potent 2' $(\beta)$ - $C$ ' methyl carbodine nucleosides. Geminal substitution at the 2'-position night impose favorable steric as well as electronic effect on the interaction with $\mathrm{HCV}$ polymerase.

As depicted in Scheme 1. we used the epoxide intermediate $\mathbf{5}$ as starting material. which could be readily syntheșized wia commercially available methylcyclopentenone 4 as described in a previous report. ${ }^{8}$ First. the hydroxy functional group was masked with a benzy'l group under the usual benzylation condi- 

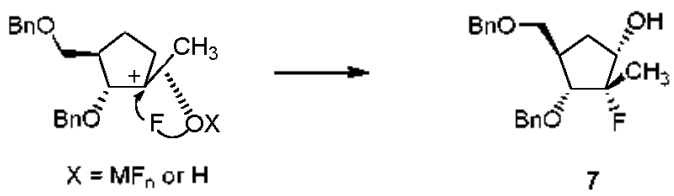

Figure 2. Possible intenmediate for the formation of 7

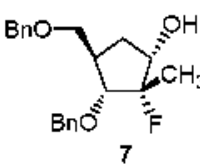<smiles>CCC1(C)[C@@H](O)C(CO)CC1(C)C</smiles>

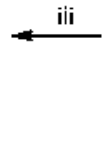<smiles>CCCCCn1ccc(NC(=O)c2ccccc2)nc1=O</smiles>

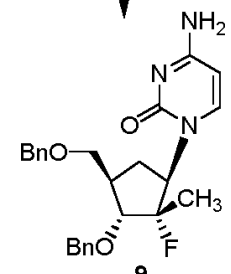

Scheme 2. Synthesis of target 2'-fluoro-cytidine analogue. Reagents: i) $\mathrm{N}^{+}$-Bz-cytosine, $\mathrm{PPl}_{3}$, DLAD; ii) $\mathrm{NaOMe} / \mathrm{MeOH}$; iii) $\mathrm{Pa}(\mathrm{OH})_{2}$, cyclohexene, $\mathrm{MeOH}$, reflux

tions (BnBr. NaH. DMF) to provide a fully protected intermediate 6, which underwent a ring-opening fluorination reaction with hydrofluoric acid in the presence of silicon fluorides and additives to provide cis-fluorolydrin in good yield (Scheme 1). ${ }^{9}$ The formation of the cis-isomer may be due to the hydrogen bonding and/or silyl ether formation as shown in Figure 2.

To synthesize the desired carbocyclic nucleoside analogues. the alcohol derivative was subjected to a Mitsunobu coupling condition. which is the most useful and common method for the direct substitution of the hydroxyl group with an inversion of the configuration. First -benzoyl cy tosine was treated with the protected fluorolydrin 7 in the presence of diisopropylazodicarboxylate (DIAD) and $\mathrm{PPh}_{3}$ to give 8 in $68 \%$ yield (Scheme 2). The removal of $\mathrm{N}^{+}$-benzoyl group of nucleoside analogue 8 was performed by sodium methoxide. Hýdrogenolysis of the benzyl protecting group of 9 with a palladium hydroxide gave the target cytosine derivative 10. For the synthesis of the adenine nucleoside analogue. similar reactions for the synthesis of the cytosine analogue were attempted. $N^{16}$-Bis-Bocadenine ${ }^{11}$ was similarly subjected to Mitsunobu coupling conditions ( $\mathrm{DIAD}, \mathrm{PPh}_{3}$ ) to give adenine analogue 11 in a high yield. 93\%. Two boc-protection groups of $\mathbf{1 1}$ were removed in trifluoric acetic acid (TFA) conditions to give 12. which was finally transformed to target compound 13 through the debenzylation conditions as used for $\mathbf{1 0}$ (Scheme 3 ).

As shown in Figure 3, the relative stereochemistry was unambiguously confirmed on the basis of the NOE results between the proximal hydrogens. On irradiation of $\mathrm{C}_{2}\left(\mathrm{CH}_{3}\right)-\mathrm{H}$. relatively weak NOE was observed at $\mathrm{C}_{\mathrm{J}}-\mathrm{H}(0.21 \%)$. compared to that of $\mathrm{C}_{3}-\mathrm{H}(0.86 \%)$.

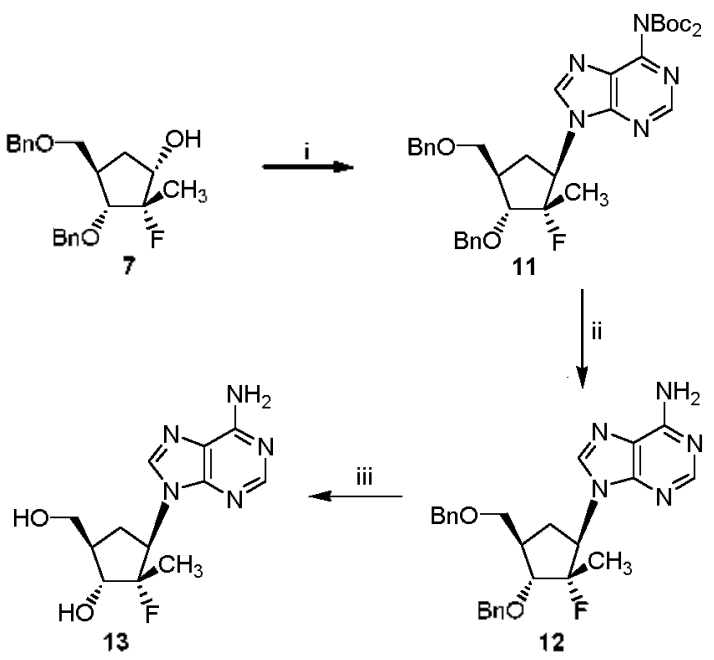

Scheme 3. Synthesis of target 2'-tluoro-adenosine analogue. Reagents: i) $\mathrm{N}^{(6}$-bis-Boc-adenirle, $\mathrm{PPh}_{3}$, DIAD, $0^{\circ} \mathrm{C}$; ii TFA, DCE/MeOH, rt; iii) $\mathrm{Pa}(\mathrm{OH})_{2}$, cyclohexene, $\mathrm{MeOH}$, reflux

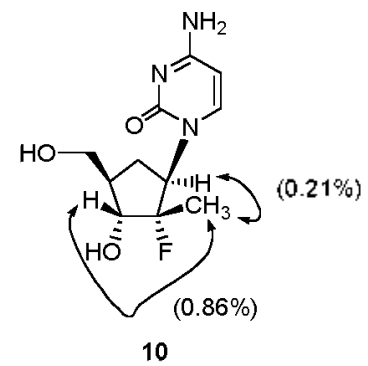

Figure 3. Possible intemediate for the fomation of 7 .

Table 1. Anti-HCV activity of the newly synthesized compounds 10 and 13

\begin{tabular}{ccc}
\hline Compound No. & $\begin{array}{c}\text { Anti-HCV } \\
\mathrm{EC}_{3(1}(\mu \mathrm{g} / \mathrm{mL})\end{array}$ & $\begin{array}{c}\text { Cytotoxicity } \\
\mathrm{C}\end{array}$ \\
\hline 10 & 18.2 & 32.1 \\
13 & $>50$ & $>50$ \\
2 '-C-Me-Cyt & 3.7 & $>50$ \\
\hline
\end{tabular}

2'-C-Me-Cyt: 2'-C-Methyloytidine. $\mathrm{EC}_{50}($ hg $\mathrm{mL}$ ): concentration required to inhibit $50^{\circ}$ of the virus induced cy topathicity. $C_{5 n}(\mu \mathrm{gmL})$ : concentration required to reduce cell viability by $\mathbf{5 0} 0$.

The sy nthesized conpounds were tested for anti-HCV activity using an in witro assay. This system is composed of a human hepatocarcinoma cell line (Huh-7) supporting multiplication of an HCV replicon named NK-R2AN. ${ }^{22}$ Cytosine analogue 10 weakly inhibited the replication of the replicon. NK-R2AN. in Huh- 7 cells by $50 \%$ at $18.2 \mu \mathrm{M}$ (Table 1 ).

In summary, the present ring-opening fluorination of epoxide using hydrofluoric acid offers a convenient procedure for the synthesis of cis-fluorhydrins. On the basis of potent anti-HCV activity of 2'-modified nucleosides, we have designed and synthesized 2' $(\alpha)$ - $C$-fluoro-2' $(\beta)-C$-methỵl carbodine derivatives from 2-methyl cyclopentenone. The cy tosine analogue 10 exhibited potent anti-HCV activity. 


\section{Experimental Section}

Melting points were determined on a Mel-temp II laboratory device and are uncorrected. NMR spectra were recorded on a JEOL JNM-AL300 Fourier transform chemical shifts are reported in parts per million $(\delta)$ and signals are reported as $s$ (singlet). $\mathrm{d}$ (doublet). $\mathrm{t}$ (triplet). q (quartet). $\mathrm{m}$ (multiplet) and dd (doublet of doublets). UV spectra were obtained on a Beckman DU-7 spectrophotometer. The elemental analyses were performed using a Perkin-Elmer 2400 analyzer. TLC was performed on Uniplates (silica gel) purchased from Analtech Co. All reactions were performed under an atmosphere of nitrogen unless specified. Dry dichloromethane. benzene. and pyridine were obtained by distillation from $\mathrm{CaH}_{2}$. Dry' THF was obtained by distillation from $\mathrm{Na}$ and benzophenone immediately prior to use

(rel)-(1S,2R,3S,5S)-2-Benzyloxy-3-benzyloxymethy]-1methyl-6-0xabicyclo[3,1,0]hexane (6). To a solution of epoxide derivative 5 ( 2.1 g. $8.96 \mathrm{mmol})$ in dry DMF (20 $\mathrm{mL})$ was slowly added $\mathrm{NaH}$ (258 $\mathrm{mg} .10 .75 \mathrm{mmol}$ ) at $0^{\circ} \mathrm{C}$. After $30 \mathrm{~min}$. benzyl bromide ( $1.68 \mathrm{~g}, 9.85 \mathrm{mmol}$ ) was added. and the reaction mixture was stirred for $3 \mathrm{~h}$ at it. The mixture was quenched by adding of saturated ammonium clloride $(2 \mathrm{~mL})$ and poured into water $(30 \mathrm{~mL})$. The mixture was extracted with ethyl acetate $(30 \mathrm{~m})$ two times. The combined organic layer was washed with brine and dried over anhydrous $\mathrm{MgSO}_{4}$. filtered, and evaporated. The filtrate was concentrated under reduced pressure and the residue was purified by silica gel column chromatography (EtOAc/hexane, 1:20) to give $6(2.29 \mathrm{~g} .79 \%$ ) as colorless oil. ${ }^{1} \mathrm{H} \mathrm{NMR}\left(\mathrm{CDCl}_{3}, 300 \mathrm{MHz}\right)$ ò 7.35-7.29 (m. 10H). 4.59 (s. 2H). 4.53 (s, 2H). 3.98 (d. $J=7.0 \mathrm{~Hz}, 1 \mathrm{H}), 3.50$ (dd. $J=5.4 .9 .2 \mathrm{~Hz}, \mathrm{lH}) .3 .34(\mathrm{dd} J=6.2 .9 .2 \mathrm{~Hz}, 1 \mathrm{H}) .2 .31(\mathrm{~m}$. 1H). $2.13(\mathrm{~m} . \mathrm{HH}) .1 .79(\mathrm{dd}, J=6.3,10.6 \mathrm{~Hz} .1 \mathrm{H}) .1 .64(\mathrm{dd}, J=$ $8.2,10.5 \mathrm{~Hz}, \mathrm{lH}), 1.36(\mathrm{~s}, 3 \mathrm{H}):{ }^{13} \mathrm{C} \mathrm{NMR}\left(\mathrm{CDCl}_{3}\right) \delta 139.2$. $138.1,128.3,127.9,127.1,126.4 .79 .4,77.2 .74 .2 .72 .6,68.8$. 60.7. 41.3. 26.4. 14.3; $\mathrm{MS}(\mathrm{FAB}+) m / z 325(\mathrm{M}+\mathrm{H})^{+}$.

(rel)-(1S,2S,3R,4S)-3-Benzyloxy-4-benzyloxymethyl-2fluon-2-methyl-cyclopentanol (7). To a mixture of $\left(\mathrm{NH}_{4}\right)_{2} \mathrm{SiF}_{6}$ (890 mg. $5.0 \mathrm{mmol}$ ). CsF ( $151.9 \mathrm{mg} .1 .0 \mathrm{mmol}$ ) and epoxide (324 mg. $1.0 \mathrm{mmol}$ ) in 1.2-dichloroethane ( $10 \mathrm{~mL}$ ) in polyethylene bottle was added $47 \%$ hydrofluoric acid $(0.127 \mathrm{~mL} .3 .0$ mmol) at $0^{\circ} \mathrm{C}$. and the mixture was stirred for $7 \mathrm{~h}$ at $0^{\circ} \mathrm{C}$. A saturated $\mathrm{NaHCO}_{3}$ solution $(10 \mathrm{~mL}$ ) was slowly added and the whole mixture was extracted with diethyl ether $(10 \mathrm{~mL})$ two times. The combined organic layer was washed with brine and dried over anlyydrous $\mathrm{MgSO}_{4}$. filtered. and evaporated. The filtrate was concentrated under vacuum and the residue was purified by silica gel column chromatography (EtOAc/hexane, $\mathrm{L}: \mathrm{l0}$ ) to give alcohol $7\left(175 \mathrm{mg} .51 \%\right.$ ) as a colorless oil. ${ }^{1} \mathrm{H}$ NMR (CDCl $300 \mathrm{MHz}) \delta 7.36-7.27(\mathrm{~m}, 10 \mathrm{H}) .4 .61(\mathrm{~s}, 2 \mathrm{H}) .4 .57$ (s. $2 \mathrm{H}) .3 .86$ (ddd $J=2.8,6.2 .18 .+\mathrm{Hz} . \mathrm{IH}) .3 .57$ (dd. $J=5.8 .9 .0$ Hz. lH) .3 .24 (dd $J=5.8 .13 .8 \mathrm{~Hz}, 1 \mathrm{H}), 2.29(\mathrm{~m} .1 \mathrm{H}) .1 .76$ (dd. $J=6.2 .10 .4 \mathrm{~Hz} .1 \mathrm{H}) .1 .59(\mathrm{dd} . J=8.4 .10 .4 \mathrm{~Hz} .1 \mathrm{H}), 1.28(\mathrm{~d}$, $J=21.8 \mathrm{~Hz} .3 \mathrm{H}):{ }^{13} \mathrm{C} \mathrm{NMR}\left(\mathrm{CDCl}_{3}\right) \dot{o} 138.8 .138 .2,130.1$. 128.4. $127.6 .127 .0 .126 .1 .102 .8(\mathrm{~d} . J=181.2 \mathrm{~Hz}), 80.2(\mathrm{~d}, J=$ $+2.6 \mathrm{~Hz}), 76.1 .75 .5 .73 .2(\mathrm{~d} J=18.8 \mathrm{~Hz}), 69.2,38.8,28.4 .14 .1$ (d, $J=24.5 \mathrm{~Hz}$ ); Anal Calcd. for $\mathrm{C}_{21} \mathrm{H}_{24} \mathrm{O}_{3}$ : C. $77.75 ; \mathrm{H} .7 .46$. Found: C. 77.82: H, 7.38; $\mathrm{MS}(\mathrm{FAB}+) m / z 345(\mathrm{M}+\mathrm{H})^{-}$: Anal. Calcd for $\mathrm{C}_{\supseteq 1} \mathrm{H}_{2 \leq} \mathrm{FO}_{3}:$ C. 73.23: H. 7.32. Found: C. 73.29: H. 7.27.
(rel)-(1R,2S,3R,+S)-1-(3-Benzyloxy-4-benzyloxymethyl2-fluoro-2-methyl-cyclopentan-1-yl) $\dot{N}^{+}$-benzoyl cytosine (8). To a stirred solution of triphenylphosphine (56l $\mathrm{mg} .2 .14 \mathrm{mmol}$ ) in dry THF ( $8 \mathrm{~mL}$ ) at $0{ }^{\circ} \mathrm{C}$ was added dropwise the diisopropyl azodicarboxylate (DIAD) (432 $\mathrm{mg} .2 .14 \mathrm{mmol}$ ) and the reaction nixture was stirred at this temperature for 30 min. After that, a solution of the alcohol 7 (368 $\mathrm{mg} .1 .07 \mathrm{mmol})$ in THF ( $8 \mathrm{~mL})$ was added and the reaction nixture was stirred at $0^{\circ} \mathrm{C}$ for 15 min. Then the cold bath was removed and the yellow solution was stirred for $30 \mathrm{~min}$ at room temperature. $N^{-1}$-Benzoyl cy tosine (460 mig. 2.14 nmol) was then added and the reaction mixture was stirred overnight at room temperature. The reaction mixture was concentrated under reduced pressure and the residue was purified by silica gel column chromatography (EtOAc/hexane. $3: 1)$ to give compound $8(401 \mathrm{mg} .68 \%)$ : $\mathrm{mp} 178-180{ }^{\circ} \mathrm{C}:{ }^{1} \mathrm{H}$ NMR $\left(\mathrm{CDCl}_{5} .300 \mathrm{MHz}\right) \delta 8.29(\mathrm{~d}, J=7.2 \mathrm{~Hz}, 1 \mathrm{H}), 7.92(\mathrm{dd}$, $J=5.6 .8 .6 \mathrm{~Hz}, 2 \mathrm{H}), 7.62(\mathrm{~d} J=5.0 \mathrm{~Hz} 2 \mathrm{H}) .7 .38-7.26(\mathrm{~m} \mathrm{llH})$, $5.60(\mathrm{~d} . J=7.2 \mathrm{~Hz} .1 \mathrm{H}), 4.63(\mathrm{~s} .2 \mathrm{H}), 4.56(\mathrm{~s}, 2 \mathrm{H}), 4.02(\mathrm{dd} . J=$ $5.8 .16 .8 \mathrm{~Hz} . \mathrm{lH}$ ) 3.54 (dd. $J=8.2 .12 .0 \mathrm{~Hz} . \mathrm{lH}$ ). 3.22 (ddd. $J=1.8,6.2,1+.6 \mathrm{~Hz} .1 \mathrm{H}), 2.30$ (m. $1 \mathrm{H}), 1.85$ (dd. $J=8.6,10.8$ Hz. $1 \mathrm{H}$ ). 1.49 (dd $J=6.2 .10 .7 \mathrm{~Hz} .1 \mathrm{H}$ ). 1.32 (d. $J=22.1 \mathrm{~Hz}$, $3 \mathrm{H}):{ }^{13} \mathrm{C} \mathrm{NMR}\left(\mathrm{CDCl}_{3}\right) \hat{\delta} 170.38,165.9,157.8,138.2,136.2$. $133.5,132.9 .129 .6 .128 .1 .127 .7 .127 .1 .126 .3,101.9(\mathrm{~d}, J=$ $181.8 \mathrm{~Hz}), 94.3,81.5(\mathrm{~d}, J=44.1 \mathrm{~Hz}), 78.2 .76 .2,68.7,55.5$ (d. $J=16.8 \mathrm{~Hz}) .36 .2 .25 .2,14.5(\mathrm{~d}, J=28.6 \mathrm{~Hz}): \mathrm{MS}(\mathrm{FAB}+)$ $\mathrm{m} / 2564(\mathrm{M}+\mathrm{Na})^{-}$

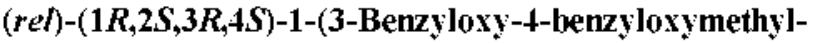
2-fluoro-2-methyl-cyclopentan-1-yl) cytosine (9). To a stirred solution of compound 8 ( $32+\mathrm{mg} .0 .6 \mathrm{mmol})$ in $\mathrm{MeOH}(8 \mathrm{~mL})$. $\mathrm{NaOMe}(0.3 \mathrm{~mL}, 1 \mathrm{M}$ solution in $\mathrm{MeOH})$ was added at $0^{\circ} \mathrm{C}$ under nitrogen and stirred overnight. The reaction mixture was neutralized with acetic acid and concentrated under reduced pressure. The residue was purified by silica gel column chromatography (EtOAc/Hexane/MeOH, 3:1:0.2) to give compound 9 (233 nig. $89 \%$ ) as a white solid: $\mathrm{mp} 169-171^{\circ} \mathrm{C}$ : UV (MeOH) $\lambda_{\max } 271.0 \mathrm{~mm}:{ }^{1} \mathrm{H}$ NMR $\left(\mathrm{CDCl}_{\underline{-} .} 300 \mathrm{MHz}\right)$ ô 7.81 (d. $J=7.2$ Hz. $1 \mathrm{H}), 7.31-7.25(\mathrm{~m}, 10 \mathrm{H}), 5.57(\mathrm{~d}, J=7.2 \mathrm{~Hz}, 1 \mathrm{H}) .4 .64(\mathrm{~s}$, $2 \mathrm{H}$ ). 4.57 (s. 2H), 4.06 (ddd. $J=2.8 .6 .4 .18 .8 \mathrm{~Hz}, \mathrm{lH}$ ), 3.59 (dd. $J=8.2 .12 .2 \mathrm{~Hz}, \mathrm{IH}), 3.27$ (dd. $J=6.4 .14 .4 \mathrm{~Hz} . \mathrm{lH}$ ). 2.34 $(\mathrm{m}, 1 \mathrm{H}) .1 .81(\mathrm{dd}, J=8.4,10.6 \mathrm{~Hz} .1 \mathrm{H}), 1.39(\mathrm{dd} . J=6.2 .10 .6$ $\mathrm{Hz}, \mathrm{IH}) .1 .37(\mathrm{~d} . J=20.8 \mathrm{~Hz}, 3 \mathrm{H}):{ }^{13} \mathrm{C}$ NMR $\left(\mathrm{CDCl}_{3}\right) \delta 165.7$. $156.5,142.5,137.8$. 136.8, 134.2. 133.2, 129.4, 128.6. 127.9. $127.2,126.9,103.1(\mathrm{~d} J=181.2 \mathrm{~Hz}) .94 .9 .83 .5(\mathrm{~d} . J=42.1 \mathrm{~Hz})$. $79.0,77.1,68.4 .56 .7$ (d. $J=17.2 \mathrm{~Hz}) .35 .8 .26 .7,14.2(\mathrm{~d}, J=$ $27.8 \mathrm{~Hz}) ; \mathrm{MS}(\mathrm{FAB}+) m / z+38(\mathrm{M}+\mathrm{H})^{+}$.

$(\mathrm{rel})-(1 R, 2 S, 3 R, 4 S)-1-(3-H y d o x y-4-h y d o x y m e t h y l-2-f l u-$ oro-2-methyl-cyclopentan-1-yl) cytosine (10). A solution of 9 (37I $\mathrm{mg} .0 .85 \mathrm{mmol})$ in $\mathrm{MeOH}(25 \mathrm{~mL})$ was treated with palladiun hydroxide $\left(170 \mathrm{mg}, 20 \% \mathrm{in}\right.$ activated clarcoal) at $0{ }^{\circ} \mathrm{C}$. Cyclohexene $(10 \mathrm{~mL})$ was added and the reaction mixture was refluxed overnight. The suspension was cooled down to room temperature. filtered over Celite, and the filtrates were concentrated. The residue was purified by silica gel column chromatography $\left(\mathrm{CH}_{2} \mathrm{Cl}_{2} / \mathrm{MeOH} .5: 1\right)$ to give compound 10 (188 mig. $86 \%$ ) as a white solid: $m p 202-204^{\circ} \mathrm{C}$ : UV $\left(\mathrm{H}_{2} \mathrm{O}\right) \mathrm{h}_{\max } 271.5$ nnt: ${ }^{1} \mathrm{H}$ NMR (300 MHz. DMSO- $d_{6}$. $\mathrm{D}_{2} \mathrm{O}$ exchanged) ô 7.87 $(\mathrm{d} . J=7.2 \mathrm{~Hz} .1 \mathrm{H}), 5.67$ (d. $J=7.2 \mathrm{~Hz}, 1 \mathrm{H}) .4 .97(\mathrm{~d}, J=5.2 \mathrm{~Hz}$. lH) 4.89 (t. $J=5.4$ Hz. lH.) 4.65 (s. $2 \mathrm{H}$ ). 4.57 (s. $2 \mathrm{H}$ ). 4.03 (dd. 
$J=6.6 .18 .6 \mathrm{~Hz}, 1 \mathrm{H}), 3.52(\mathrm{dd} . J=8.0 .10 .8 \mathrm{~Hz}, 1 \mathrm{H}), 3.31(\mathrm{dd}$. $J=6.6,14.8 \mathrm{~Hz}, 1 \mathrm{H}) .2 .36(\mathrm{~m} .1 \mathrm{H}) .1 .83(\mathrm{dd}, J=8.6,11.2 \mathrm{~Hz}$. IH) 1.39 (dd $J=6.4 .11 .2 \mathrm{~Hz}, 1 \mathrm{H}) .1 .39($ d. $J=21.6 \mathrm{~Hz}, 3 \mathrm{H})$; ${ }^{13} \mathrm{C} \mathrm{NMR}$ (DMSO- $d_{6}$. $\mathrm{D}_{2} \mathrm{O}$ exchanged) $\delta 165.8,156.3 .143 .6$, 101.9 (d. $J=182.0 \mathrm{~Hz}) .94 .5 .84 .1(\mathrm{~d} . J=40.8 \mathrm{~Hz}$ ). 78.9 .76 .3 . $68.8,55.8(\mathrm{~d}, J=16.8 \mathrm{~Hz}) .36 .5 .25 .3,13.8(\mathrm{~d} . J=26.6 \mathrm{~Hz}) ; \mathrm{MS}$ $(\mathrm{FAB}+) m / 2280(\mathrm{M}+\mathrm{Na})^{-}$: Anal. Calcd for $\mathrm{C}_{13} \mathrm{H}_{16} \mathrm{FN}_{3} \mathrm{O}_{3}$ $(+0.5 \mathrm{MeOH}):$ C. 50.54: H, 6.64: N, 16.38. Found: C, 50.46: $\mathrm{H}, 6.59: \mathrm{N}, 16.32$

(rel)-(1R,2S,3R,4S)-9-(3-Benzyloxy-4-benzyloxymethyl2-fluon-2-methyl-cyclopentan-1-yl) $N^{6}$-bis-Boc-adenine (11). Nucleoside analogie 11 was synthesized from $N^{6}$-bis-Boc-protected adenine by the same procedure as described for the preparation of 8 : yield $93 \%:{ }^{l} \mathrm{H}$ NMR $\left(\mathrm{CDCl}_{3} .300 \mathrm{MHz}\right.$ ) ò 8.82 (s. lH) $7.97(\mathrm{~s} . \mathrm{lH}) .7 .32-7.2+(\mathrm{m} .10 \mathrm{H}) .4 .66(\mathrm{~s} .2 \mathrm{H}) .4 .58(\mathrm{~s} .2 \mathrm{H})$. 4.07 (ddd $J=2.0,6.8 .15 .6 \mathrm{~Hz} . \mathrm{HH}), 3.58(\mathrm{dd}, J=8.4 .12 .2 \mathrm{~Hz}$, $\mathrm{IH}) .3 .38(\mathrm{dd} J=6.8 .15 .2 \mathrm{~Hz}, \mathrm{IH}) .2 .34(\mathrm{~m}, \mathrm{IH}) .1 .82(\mathrm{dd} J=$ $8.8,12.2 \mathrm{~Hz}, 1 \mathrm{H}) .1 .54(\mathrm{dd}, J=6.6,12.2 \mathrm{~Hz}, 1 \mathrm{H}), 1.43(\mathrm{~s}, 18 \mathrm{H})$, $1.33(\mathrm{~d} J=20.8 \mathrm{~Hz}, 3 \mathrm{H}){ }^{13} \mathrm{C} \mathrm{NMR}\left(\mathrm{CDCl}_{3}\right)$ ò $153.1,152.8$. $152.1,150.4 .142 .4,138.5$. 137.5. 134.2, 133.6. 129.1, 128.2, $127.8,127.2,119.4 .104 .9(\mathrm{~d}, J=182.2 \mathrm{~Hz}), 83.6,79.5(\mathrm{~d}, J=$ $40.8 \mathrm{~Hz}) .77 .8 .76 .1,67.9 .54 .3(\mathrm{~d} . J=17.6 \mathrm{~Hz}), 37.4 .27 .5 .26 .7$. $14.1(\mathrm{~d}, J=26.4 \mathrm{~Hz}): \mathrm{MS}(\mathrm{FAB}+) m / z 684(\mathrm{M}+\mathrm{Na})^{+}$.

(rel)-(1R,2S,3R,4S)-9-(3-Benzyloxy-4-benzyloxymethyl2-fluoro-2-methyl-cyclopentan-1-yl) adenine (12). To a stirred solution of $11(+10 \mathrm{mg}, 0.62 \mathrm{mmol})$ in $\mathrm{ClCH}_{2} \mathrm{CH}_{2} \mathrm{Cl} / \mathrm{MeOH}=$ $1: 1(5 \mathrm{~mL})$ was added dropwise trifluoric acid $(2.6 \mathrm{~g} .23 .1 \mathrm{mmol})$ and the reaction mixture was stirred at room temperature overnight. The mixture was concentrated under reduced pressure and the residue was purified by silica gel column chromatography (EtOAc/Hexane/MeOH. 3:1:0.1) to give compound 12 (263 $\mathrm{mg} .92 \%$ ) as a white solid: $\mathrm{mp} 200-202^{\circ} \mathrm{C}$ : UV (MeOH) $\lambda_{\text {max }} 259.5 \mathrm{~nm} ;{ }^{l} \mathrm{H} \mathrm{NMR}\left(\mathrm{CDCl}_{3}, 300 \mathrm{MHz}\right)$ ò 8.36 (s. $\left.1 \mathrm{H}\right), 8.28$ (s. $1 \mathrm{H}) .7 .36-7.28(\mathrm{~m}, 10 \mathrm{H}), 4.61$ (s. $2 \mathrm{H}), 4.52$ (s. $2 \mathrm{H}), 4.01$ (dd. $J=6.4 .15 .8 \mathrm{~Hz}, 1 \mathrm{H}), 3.52$ (dd. $J=8.6 .12 .0 \mathrm{~Hz}, 1 \mathrm{H}), 3.40$ (dd. $J=7.0 .15 .8 \mathrm{~Hz}, 1 \mathrm{H}) .2 .37(\mathrm{~m} .1 \mathrm{H}), 1.79(\mathrm{dd}, J=8.6,12.0 \mathrm{~Hz}$. $1 \mathrm{H}), 1.56(\mathrm{dd}, J=6.4 .12 .1 \mathrm{~Hz}, 1 \mathrm{H}) .1 .35(\mathrm{~d} . J=21.2 \mathrm{~Hz}, 3 \mathrm{H})$; ${ }^{13} \mathrm{C} \mathrm{NMR}\left(\mathrm{CDCl}_{3}\right)$ o $155.6,152.2,150.1 .149 .4,137.8,136.4$. $134.5,132.2,129.4,128.1,127.2,119.6 .101 .5$ (d. $J=180.8 \mathrm{~Hz})$. $80.1(\mathrm{~d} J=44.3 \mathrm{~Hz}) .78 .2 .76 .5 .68 .5 .56 .7(\mathrm{~d} . J=18.8 \mathrm{~Hz}), 38.3$, 27. 1. $14.7(\mathrm{~d}, J=25.8 \mathrm{~Hz})$ : $\mathrm{MS}(\mathrm{FAB}+) m / z+8+(\mathrm{M}+\mathrm{Na})^{+}$.

(rel)-(1R,2S,3R,4S)-9-(3-Hydroxy-4-hydroxymethyl-2-fluoro-2-methyl-cyclopentan-1-yl) adenine (13). Adenine analogue 13 was obtained by a similar procedure as described for the preparation of 10 : yield $84 \% ;$ mp $216-219^{\circ} \mathrm{C}$; UV $\left(\mathrm{H}_{2} \mathrm{O}\right) \lambda_{\text {max }}$ 260. nm: ${ }^{1} \mathrm{H}$ NMR (300 MHz. DMSO- $d_{6} . \mathrm{D}_{3} \mathrm{O}$ exchanged) $\tilde{o}$ $8.32(\mathrm{~s}, 1 \mathrm{H}), 8.2+(\mathrm{s}, 1 \mathrm{H}), 4.62$ (s. $2 \mathrm{H}) .4 .55$ (s. $2 \mathrm{H}) .4 .00(\mathrm{dd}$, $J=6.2 .15 .4 \mathrm{~Hz}, \mathrm{IH}) .3 .5 \mathrm{l}(\mathrm{dd} . J=8.8 .12 .2 \mathrm{~Hz}, \mathrm{IH}) .3 .39(\mathrm{dd}$. $J=7.2,15.6 \mathrm{~Hz}, 1 \mathrm{H}) .2 .35(\mathrm{~m}, 1 \mathrm{H}) .1 .80(\mathrm{dd}, J=8.8,12.0 \mathrm{~Hz}$, IH) $1.58(\mathrm{dd} J=6.6 .12 .0 \mathrm{~Hz}, 1 \mathrm{H}) .1 .37(\mathrm{~d} . J=21.0 \mathrm{~Hz}, 3 \mathrm{H})$;
${ }^{12} \mathrm{C}$ NMR (DMSO- $d_{6}, \mathrm{D}_{2} \mathrm{O}$ exchanged) ô $155.7,153.2 .150 .2$, $148.3,120.0,103.6$ (d. $J=182.2 \mathrm{~Hz}) .81 .2(\mathrm{~d} J=44.6 \mathrm{~Hz}), 79.1$. $75.2 .68 .8 .55 .3(\mathrm{~d} J=20.2 \mathrm{~Hz}), 37.1 .26 .7 .14 . \mathrm{I}(\mathrm{d} J=24.6 \mathrm{~Hz})$ : $\mathrm{MS}(\mathrm{FAB}+) m / 2304(\mathrm{M}+\mathrm{Na})^{-}$: Anal. Calcd for $\mathrm{C}_{12} \mathrm{H}_{1} \mathrm{FN}_{5} \mathrm{O}_{2}$ $\left(+1.0 \mathrm{H}_{2} \mathrm{O}\right): \mathrm{C} .48 .15: \mathrm{H}, 6.06: \mathrm{N}, 23.40$. Found: C. 48.19: H, 5.97: N, 23.36.

Acknowledgments. This work was supported by the Pioneer Research Program for Converging Technology of the Ministry of Education, Science and Technology, Republic of Korea (Grant No. M1071118001-08M1118-00110) (2008).

\section{Reference}

1. Tan, S. L.: Panse, A.; Shi, Y.; Sonenberg, N. Nat. Rev. Dnng Discoverv 2002, $1,867-881$.

2. Heathcote, E.; Shiffman, M.; Cooksley, W; Dusheiko, G. M: Lee, S. S.: Balart, L.: Reindollar, R. Reddy, R. K.: Wright, T. L.; Lin, A.; Hoftman, T.: De Pamphilis, J. N. Engl. J.A ked 2000, 343, 16731680 .

3. (a) Clark, J. L.; Hollecker, L.; Mason, J. C.; Stuyver L. J.; Thanish, P. M.; Lostia, S: McBraver, T. R.; Schinazi, R. F.; Watanabe, K. A.; Otto, M. J.; Furman, P. A.; Stec, W. T.; Patterson, S. E.: Pankiewicz. K. W. J. Med. Chem 2005, 48,5504-5508 (b) Smith. D. B. Kalay anov, G.; Sund, C.; Winquist, A.; Pinho, P.; Maltseva, T:; Morisson, V.; Leveque, V.; Rajyaguru, S.; Le Pogam, S.; Najera, I.; Benkestock, K; Zhou, X. X.; Maag. H.; Cammack, N.; Martin, J. A.: Swallow, S., Tohansson, N. G.: Klumpp, K, Smith, M. J. Wed. Chem. 2009, 52, 219-223: (c) Li, H.; Baik, Y. C.; Hong, J. H. Bull. Kowem Chem. Soc. 2009, $30,1147-1151$.

4. El Kouni, M. H. Curn: Phamt Des. 2002, 8, 581-593

5. Carrol, S. S.: Tomassini, J. E.: Bossenman, M: Getty, K.: Stahlhut, M. W.; Eldrup, A. B.: Bhat, B.; Hall, D.; Simcoe, A. L.: LaFemina, R.; Rutkowski, C. A.; Wolanski, B.; Yang, Z.; Migliaccio, G.; De Francesco, R.; Kuo, L. C.; MacCross, M.; Olsen, D. B. J. Biol. Chem. 2003, 278, $11979-11984$

6. Eldrup, A. B.: Allerson, C. R.: Bennett, C. F.; Bera, S.; Bhat, B.; Bhat, N.; Bosseman, M. R.; Brooks, J.; Burlein, C.; Carrol, S. S.; Cook, P. D; Getty, K. L.; MacCross, M.; McMasters, D. R.; Olsen, D. B.: Prakash, T. P.; Prhave, M.: Song, Q. L.; Tomassini, T. E.: Xia. I. J. Wed Chem 2004, 47, 2283-2295.

7. (a) Borthwick, A. D.: Biggadike, K. Tewahedron 1992, 48, 571623: (b) Huryt D. M.: Okabe, M. Chem. Rev. 1992, 92, 1745-1768; (c) Agrofoglio, L.; Suhas, E; Farese, A.; Condom, R.; Challand, S.; Earl, R. A.; Guedj, R. Tetrahedron 1994, 50, 1061 1-10670; (d) Crimmins, M. T. Tetrahedron 1998, 54, 9229-9272: (e) Ariona, O.; Gómez, A. M.; López, T. C.; Plumet, J. Chem. Rev. 2007, 107, 1919-2036

8. Gosselin, G.: Criffe, L.; Meillon, J-C.: Storer, R. Tetrahedron 2006, 62, 906-914

9. Shimizu, M.; Nakahara, Y. J. Fluorine Chem. 1999, 99, 95-97.

10. Kitade, Y.; Ando, T.; Yamaguchi, T.; Hori, A.; Nakanishi, M.; Ueno, Y. Bioorg. Wed. Chem. 2006, $14,5578-5583$.

11. Michel, B. Y.; Strazewski, P. Tetrahedron 2007, 63, 9836-9841.

12. Kim, H. J.: Sharon, A.; Bal, C.; Wang, J.: Allu, M.; Huang, Z: Murray, M. G.; Bsaait, L.; Schinazi, R. F.; Korba, B.; Chu, C. K. J. Hed. Chent. 2009, $52,206-213$. 